\title{
Clinical features of asthma with comorbid bronchiectasis: a systematic review and meta-analysis.
}

\author{
shiqi zhang ${ }^{1}$, xiaofeng xiong ${ }^{1}$, zuohong $\mathrm{wu}^{1}$, tingting huang ${ }^{1}$, and deyun cheng $^{1}$
}

${ }^{1}$ Affiliation not available

May 19, 2020

\begin{abstract}
Objective: This meta-analysis aimed to systematically estimate the prevalence of comorbid bronchiectasis in patients with asthma and to summarize its clinical impact. Data sources: Embase, PubMed, and Cochrane Library electronic databases were searched to identify relevant studies published from inception until March 2020. Study Selection: Studies were included if bronchiectasis was identified by high-resolution computed tomography. Outcomes included prevalence of bronchiectasis and its association with demographic characteristics and indicators of asthma severity, including results of lung function tests and number of exacerbations. Results: Five observational studies with 839 patients were included. Overall, the mean prevalence of bronchiectasis in patients with asthma was 36.6\% (307/839). Patients with comorbid bronchiectasis had lower forced expiratory volume (FEV1)/forced vital capacity (FVC) (MD: -2.71; 95\% CI: -3.72 to -1.69) and more frequent exacerbations (MD: 0.68; 95\% CI: 0.03 to 1.33) than those with asthma alone, there was no significant difference of sex, duration of asthma and serum levels of immunoglobulin(Ig)Es between asthmatic patients with or without bronchiectasis. Conclusion: The presence of bronchiectasis in patients with asthma was associated with greater asthma severity. There are important therapeutic implications of identifying bronchiectasis in asthmatic patients.
\end{abstract}

\section{Clinical features of asthma withcomorbid bronchiectasis: a systematic review and meta-} analysis

Running head: Clinical features of comorbid asthma and bronchiectasis

Author information

Shi-qi Zhang ${ }^{1}$, Xiao-feng Xiong ${ }^{1}$, Zuo-hong $\mathrm{Wu}^{1}$, Ting-ting Huang ${ }^{1}$, De-yun $\mathrm{Cheng}^{1}{ }^{*}$

${ }^{1}$ Department of Respiratory and Critical Care Medicine, West China Hospital, Sichuan University, Chengdu 610041, China

*Corresponding author : De-Yun Cheng (Doctor's degree, professor of medicine), Email:chengdeyunscu@163.com

Address: NO. 37 Guoxue Alley, Chengdu, Sichuan, 610041, China

Tel: +8602885423331

First author: Shi-qi Zhang (Master's degree).

Second author: Xiao-feng Xiong (Doctor's degree).

Third authors: Zuo-hong Wu (Doctor's degree), Ting-ting Huang (Master's degree) .

Declaration of Interest: 
Funding: This study did not receive any funding.

Conflict of interest: The authors declare that they have no conflicts of interest.

Compliance with ethical standards

Research involving Human Participants: For this type of study formal consent is not required.

Informed consent: Not applicable.

\section{Acknowledgments:}

We are grateful to the authors of the primary studies included in this meta-analysis.

\section{Author Contributions}

Shi-qi Zhang and Xiao-Feng Xiong performed the literature searches, selected the studies, analyzed the data and wrote the manuscript draft. Ting-ting Huang and Zuo-hong Wu aided in the data analysis. De-Yun Cheng designed the study and revised manuscript.

\section{Hosted file}

manuscript.docx available at https://authorea.com/users/323886/articles/452327-clinicalfeatures-of-asthma-with-comorbid-bronchiectasis-a-systematic-review-and-meta-analysis

\section{Hosted file}

fig..docx available at https://authorea.com/users/323886/articles/452327-clinical-featuresof-asthma-with-comorbid-bronchiectasis-a-systematic-review-and-meta-analysis 